\title{
How I Treat Medulloblastoma in Children
}

\begin{abstract}
Medulloblastoma (MB) is the most common malignant tumor of the central nervous system in children with up to a third of these tumors presenting in children under 3 years of age. Its exquisite radio and chemosensitivity renders high cure rates in children in whom optimal resection has been achieved. Optimal surgery followed by radiation alone can cure about half of these children. The addition of chemotherapy has improved the outcomes dramatically and over $70 \%$ of children over 3 years of age with optimal resection and no metastasis can expect to be cured. Increasingly, the focus is on limiting the long-term sequelae of treatment. Precise molecular characterization can enable us to identify patients who can achieve optimal outcomes even in the absence of radiation. Insights into disease biology and molecular characterization have led to dramatic changes in our understanding, risk stratification, prognostication, and treatment approach in these children. In India, there is limited access to molecular profiling, making it challenging to apply biology driven approach to treatment in each child with MB. The Indian Society of Neuro-Oncology guidelines and the SIOP PODC adapted treatment recommendations for standard-risk MB based on the current evidence and logistic realities of low-middle income countries are a useful adjunct to guide clinical practice on a day-to-day basis in our setting.
\end{abstract}

Keywords: Childhood, craniospinal irradiation, medulloblastoma, molecular characterization

\section{Introduction}

Medulloblastoma (MB) is the most common embryonal tumour in children. The mean age at presentation is 9 years, though up to a third of these tumors present in children under 3 years of age. The predominant symptoms are secondary to obstructive hydrocephalus. Patients may also present with ataxia, cranial neuropathies or nerve root/spinal cord compression. The symptoms may be gradually progressive over weeks to months. $10 \%-40 \%$ of patients have central nervous system dissemination at diagnosis, with infants having the highest incidence. Imaging of the entire craniospinal axis is, therefore, vital at initial evaluation.

There have been major strides in the understanding of the biology and molecular characterisation of $\mathrm{MB}$ which are now being used to personalize management. Conventionally, MB has been classified based on histology (classic, desmoplastic/ nodular [DNMB], MB with extensive nodularity [MBEN], large cell/anaplastic). Besides, MB is currently classified into four

This is an open access journal, and articles are distributed under the terms of the Creative Commons Attribution-NonCommercial-ShareAlike 4.0 License, which allows others to remix, tweak, and build upon the work non-commercially, as long as appropriate credit is given and the new creations are licensed under the identical terms.

For reprints contact: WKHLRPMedknow_reprints@wolterskluwer.com distinct molecular subgroups according to transcriptional profiling studies ${ }^{[1]}$ - WNT, sonic hedgehog (SHH), Group 3, and Group 4 with prognostic implications. The molecular profiling correlates both with clinical outcomes and histologic appearance. The World Health Organization 2016 guidelines classify MB based on histology and genetically. ${ }^{[2,3]}$ Table 1 depicts the current classification.

The Indian Society of Neuro-Oncology guidelines and the SIOP-PODC guidelines for $\mathrm{MB}$ provide a pragmatic approach to the management of this condition in our setting. ${ }^{[4,5]}$

\section{Case 1}

Standard risk medulloblastoma in $>3$ years old

A 5-year-old boy presented with headaches and clumsiness starting 3 months prior to presentation. Headaches were worse in the morning and relieved by vomiting. Ophthalmic review revealed papilledema and nystagmus. His development had been normal prior to these symptoms.

Physical examination revealed ataxic gait. Urgent neuroimaging with magnetic

\footnotetext{
How to cite this article: Mahajan A. How I treat medulloblastoma in children. Indian $\mathrm{J}$ Med Paediatr Oncol 2020;41:577-86.
}

\section{Amita Mahajan}

Department of Pediatric Hematology and Oncology, Indraprastha Apollo Hospital, New Delhi, India

Submitted: 05-Apr-2020

Revised: 01-Aug-2020

Accepted: 09-Aug-2020

Published: 29-Aug-2020

Address for correspondence: Dr. Amita Mahajan, Indraprastha Apollo Hospital, New Delhi, India. E-mail: mahajanamita1@gmail. com

Access this article online Website: www.ijmpo.org DOI: 10.4103/ijmpo.ijmpo_136_20 Quick Response Code:

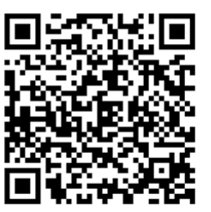


resonance imaging (MRI) [Figure 1] revealed a mass lesion in the region of the $4^{\text {th }}$ ventricle with hydrocephalus. Screening MRI of the spine revealed no evidence of leptomeningeal disease. He underwent midline suboccipital craniotomy, external ventricular drainage (EVD), and tumor resection. Histopathology confirmed classical MB with subsequent IHC-based assignation into the non-WNT, non-SHH subgroup.

Postoperative imaging [Figure 2] did not demonstrate any hemorrhage and confirmed near-total resection (NTR).

Three weeks postoperatively, lumbar puncture was done for cytopathological evaluation and was negative for malignant cells. The patient was commenced on adjuvant radiation 54 Gy with 23.4 Gy to the craniospinal axis. Radiotherapy could be completed without undue interruptions and delay.

Reassessment MRI performed 4 weeks after completion of radiation revealed no evidence of residual disease. Baseline neurological, endocrine, and auditory evaluations were performed. Adjuvant chemotherapy was commenced

\begin{tabular}{l}
\hline \multicolumn{1}{c}{ Table 1: Classification of medulloblastoma } \\
\hline Medulloblastoma genetically defined \\
Medulloblastoma, WNT-activated \\
Medulloblastoma, SHH-activated and TP53-mutant \\
Medulloblastoma, SHH-activated and TP53-wild-type \\
Medulloblastoma, non-WNT/non-SHH \\
Medulloblastoma, Group 3 \\
Medulloblastoma, Group 4 \\
Medulloblastoma, histologically defined \\
Medulloblastoma, classic \\
Medulloblastoma, desmoplastic/nodular \\
MBEN \\
Medulloblastoma, large cell/anaplastic \\
Medulloblastoma, NOS \\
NOS: Not otherwise specified, MBEN: Medulloblastoma with \\
extensive nodularity, SHH: Sonic hedgehog
\end{tabular}

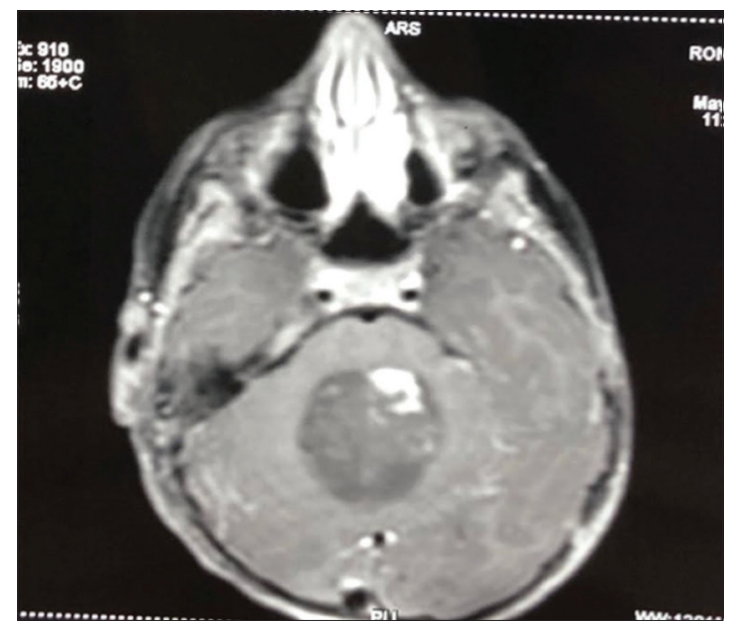

Figure 1: Standard risk medulloblastoma in $>3$ years old with vincristine, cyclophosphamide, and cisplatin. A total of six cycles were delivered. The patient continues on surveillance with MRI brain every 3 months in the $1^{\text {st }}$ year and screening MRI of the spine every 6 months. Growth, development, and neurocognitive functioning is assessed every 6 months.

Optimal management of $\mathrm{MB}$ in the current era mandates precise risk stratification incorporating stage, postsurgical status, histology, and molecular characterization.

\section{Diagnosis and staging}

The diagnosis is established with a contrast-enhanced MRI. Ideally, the entire neuraxis should be evaluated prior to surgery. Postoperative imaging may be confounded by artifacts especially blood. All patients must undergo cerebrospinal fluid (CSF) analysis 2-3 weeks after surgery. Up to $10 \%$ of patients may have malignant cells in CSF in the absence of radiological evidence of leptomeningeal dissemination. CSF analysis done earlier than 2 weeks postoperatively may be misleading. Bone marrow examination or bone scans are not routinely indicated at diagnosis unless patients are symptomatic.

The tumor extent is defined based on the presence and extent of neural and extraneural metastasis [Table 2]. The extent of resection is the most significant factor affecting prognosis. This is defined on the basis of postoperative residual disease [Table 3]. Recent evidence, ${ }^{[6]}$ however, indicates that the prognostic benefit of increased extent of resection depends on the molecular subgrouping. Maximal safe surgical resection continues to be the standard of care but repeat surgery to resect small residual portions of $\mathrm{MB}$ is not recommended if the likelihood of neurological morbidity is high. There is no definitive benefit to gross total resection compared with NTR. Specifically for patients with WNT, SHH, or Group 3 tumors, there is no significant survival benefit with gross total resection versus subtotal resection. For Group 4 tumors, gross total resection confers better progression-free survival.

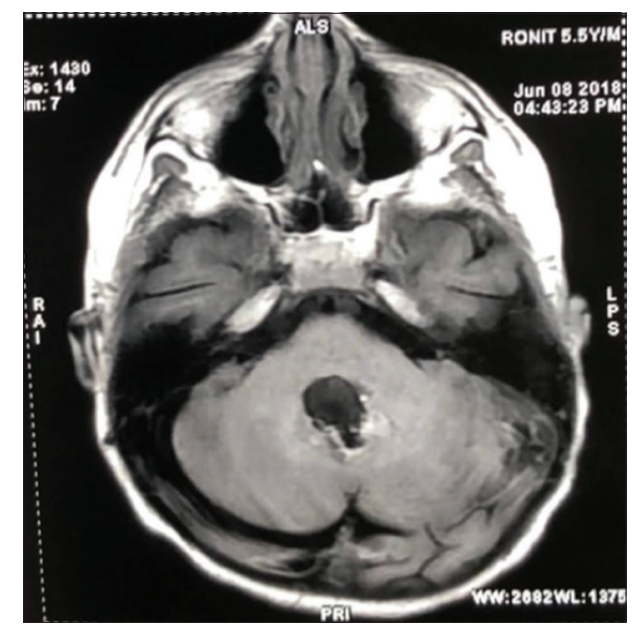

Figure 2: Standard risk medulloblastoma-postoperative 


$\frac{\text { Table 2: Staging of medulloblastoma }}{\mathrm{M}_{0}: \text { No dissemination }}$
$\mathrm{M}_{1}$ : CSF-positive cytology only
$\mathrm{M}_{2}$ : Gross nodular seeding in cerebellar-cerebral subarachnoid
space and/or lateral or third ventricle
$\mathrm{M}_{3}$ : Gross nodular seeding in spinal subarachnoid space
$\mathrm{M}_{4}$ : Extraneural metastasis

CSF: Cerebrospinal fluid

\begin{tabular}{l}
$\begin{array}{l}\text { Table 3: Classification based on postoperative residual } \\
\text { disease }\end{array}$ \\
\hline GTR: No radiographical evidence of disease \\
NTR: $\leq 1.5 \mathrm{~cm}^{2}$ residual disease after resection \\
STR: $>1.5 \mathrm{~cm}^{2}$ of measurable residual disease \\
Biopsy: No tumor resection; only a sample of tumor tissue \\
removed
\end{tabular}

STR: Subtotal resection, GTR: Gross-total resection, NTR: Neartotal resection

\section{Genetics}

Genomic profiling currently identifies four distinct subgroups WNT, SHH, Group 3, and Group 4. These molecular subtypes may further be classified based on the presence of MYC or MYCN alterations, TP53, and other genomic alterations. ${ }^{[7,8]}$

The WNT and SHH classifications define the oncopathogenic pathway, while Groups 3 and 4 retain generic designations. WNT tumors are a result of unregulated WNT signalling leading to increased transcriptional activity and tumorigenesis. WNT MB lacking somatic CTNNB1 mutation should ideally be tested for germline APC mutations (familial adenomatous polyposis syndrome) as they have significantly lower long-term survival due to deaths from second tumors, which may potentially be improved with diagnosis and surveillance. ${ }^{[9]}$

Oncogenesis in SHH MBs results from up regulation of SHH signaling as a direct consequence of loss of function of the tumor suppressor of fused gene (SUFU) and patched-1 (PTCH1). Young children with SHH MB should be screened for germline PTCH1 and SUFU and older children with TP53 mutations for Li-Fraumeni syndrome. ${ }^{[9]}$

Children with MB with WNT pathway activation have an excellent prognosis. ${ }^{[10]}$ The prognosis of patients with $\mathrm{SHH}$ pathway-activated tumors is influenced by the presence or absence of TP53 mutations. ${ }^{[1]}$ The outcome for the remaining patients is inferior to that for patients with WNT pathway activation.

WNT, SHH, Group 3, and Group 4 MBs account for 10\%, $30 \%, 25 \%$, and $35 \%$, respectively. WNT tumors are usually seen in children and adults, Group 3 tumors are more often seen in infants and children whilst SHH and Group 4 lesions are seen across all age groups. The SHH tumors exhibit a bimodal age distribution, typically occurring in patients $<4$ and $>16$ years of age. The biological behavior of SHH MB depends on the age at diagnosis with implications both on management and prognosis. ${ }^{[12]}$

While having immense prognostic and therapeutic implications, molecular profiling using genome-wide expression profiling studies is not routinely available to the vast majority of patients in low-middle income countries (LMIC). However, in experienced hands, immunohistochemistry based studies can classify the tumors into WNT, SHH, and non-WNT/non-SHH subgroups. Although it is not possible to differentiate between Groups 3 and 4 using this approach, it still offers a pragmatic approach to classify these tumours with therapeutic and prognostic implications. ${ }^{[13]}$

The role of radiogenomics in the evaluation of $\mathrm{MB}$ is evolving and may especially be valuable in LMIC settings. For example, cerebellar hemispheric tumor is likely to be SHH and a midline tumor without significant enhancement is likely to be subgroup $4 \mathrm{MB}^{[14,15]}$ In addition, many centers in LMIC do have access to FISH studies for MYC, MYCN, and Sanger seqencing for TP53. These, together with IHC and radiology, may help in risk stratification.

\section{Histologic correlation}

Although not absolute, there is a correlation between the molecular subgroup and histologic type, for example, 97\% of WNT MBs are of the classic histologic variant. In infants, children, and adults, $89 \%, 25 \%$, and $100 \%$ of DNMBs were of the SHH molecular subgroup, respectively. LC/A tumors in infants are usually Group 3 lesions, but are evenly distributed across molecular subgroups in other ages. ${ }^{[16]}$

\section{Risk stratification}

Risk assignation for children older than 3 years of age into average and high-risk prognostic groups is based on the presence of metastatic disease and residual tumor postresection of less or greater than $1.5 \mathrm{~cm}^{2}$. Patients having postoperative residual tumor $>1.5 \mathrm{~cm}^{2}$, evidence of radiographic metastases, or presence of leptomeningeal disease/CSF seeding are classified as "High-risk," with the remaining patients defined as "average-risk." ${ }^{\text {"2] }}$ Children less than 3 years of age constitute a unique group in which current standard of care is chemotherapy alone as a first-line adjunct therapy, with radiation therapy (RT) omitted to avoid the very poor neurocognitive outcomes associated with craniospinal irradiation (CSI) in very young patients.

Given the poor outcomes in patients with diffuse anaplasia, ${ }^{[17,18]}$ it is also recommended that patients with LC/A histology be classified as high risk, irrespective of other adverse features. The current consensus guidelines ${ }^{[2]}$ suggest integrating molecular subgrouping, clinical and radiological features into low risk, standard risk, high risk, and very high-risk categories with distinct survival outcomes [Table 4]. 


\begin{tabular}{|c|c|c|c|c|c|}
\hline Risk category & WNT & SHH & Group 3 & Group 4 & Others \\
\hline $\begin{array}{l}\text { Low risk } \\
\text { (Expected survival } \\
>90 \% \text { ) }\end{array}$ & $<16$ years & & & & \\
\hline $\begin{array}{l}\text { Standard risk } \\
\text { (Expected survival } \\
75-90 \% \text { ) }\end{array}$ & & $\begin{array}{l}\text { TP53 wildtype } \\
\text { No myc amplification } \\
\text { No metastasis }\end{array}$ & $\begin{array}{l}\text { No myc amplification and } \\
\text { Non-metastatic }\end{array}$ & $\begin{array}{l}\text { Non-metastatic with } \\
\text { Chromosome } 11 \text { loss }\end{array}$ & \\
\hline $\begin{array}{l}\text { High risk } \\
\text { (Expected survival } \\
50-75 \% \text { ) }\end{array}$ & & $\begin{array}{l}\text { One or both: } \\
\text { Myc amplification } \\
\text { Metastatic }\end{array}$ & & $\begin{array}{l}\text { Non-metastatic without } \\
\text { Chromosome } 11 \text { loss }\end{array}$ & \\
\hline $\begin{array}{l}\text { Very high risk } \\
\text { (Expected survival } \\
<\%)\end{array}$ & Metastatic & $\begin{array}{l}\text { TP53 mutation } \\
\text { (Metastatic or } \\
\text { non-metastatic) }\end{array}$ & Metastatic & Metastatic & \\
\hline Unknown & & & $\begin{array}{l}\text { Non-metastatic with MYC } \\
\text { amplification; anaplasia; } \\
\text { isochromosome } 17 \mathrm{q}\end{array}$ & Anaplasia & $\begin{array}{l}\text { Melanotic } \\
\text { medulloblastoma } \\
\text { Medullomyoblastoma } \\
\text { Indeterminate between } \\
\text { group } 3 \text { and } 4\end{array}$ \\
\hline
\end{tabular}

\section{Case 2}

High-risk medulloblastoma in $>3$ years old

An 8-year-old boy presented with headache, vomiting, ataxia, and head tilt for 2 weeks. Neuroimaging revealed a well circumscribed lesion $4 \mathrm{~cm} \times 4 \mathrm{~cm} \times 3.8 \mathrm{~cm}$ in the region of vermis [Figure 3].

The patient was taken up for surgery. He underwent suboccipital craniotomy with transvermian approach splitting the inferior aspect of the vermis. The tumor was highly vascular and noted to be involving the floor of the fourth ventricle, bilateral foramina of Luschka, and left cerebellar peduncle. Immediate postoperative imaging was not feasible. The patient underwent repeat MRI, 3 weeks postoperatively which revealed residual tumor of $2.5 \mathrm{cc}$. MRI of the spine demonstrated two focal enhancing nodules in the cervical and thoracic spine. CSF was positive for malignant cells. Molecular profiling confirmed it to be Group $3 \mathrm{MB}$ with myc-amplification.

He received CSI 35 Gy in 21 fractions with posterior fossa boost of 19.8 Gy with concurrent daily carboplatin. He was commenced on chemotherapy 4 weeks' following completion of radiation with vincristine/cisplatin/CCNU and cyclophosphamide. Figure 4 MRI post radiation in child with STR.

\section{Surgical management}

Optimal surgery is the cornerstone of management. The extent of resection largely depends on the anatomy of the tumor determining what can be done safely without incurring significant neurological deficit. Many studies support a relationship of extent of resection with progression-free survival. A retrospective analysis of 233 children in a randomized controlled trial of differing

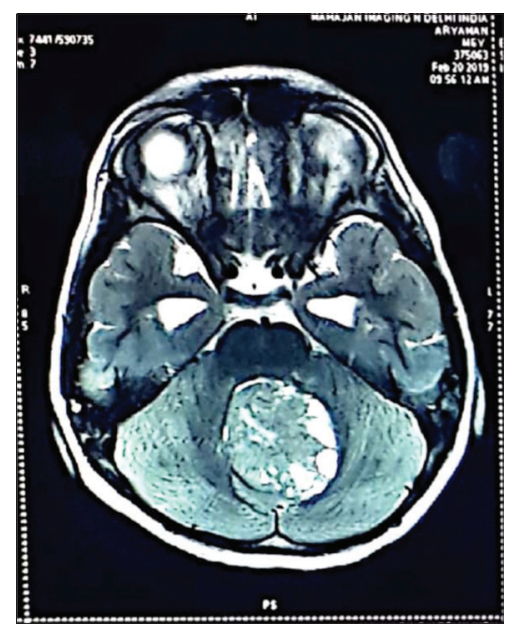

Figure 3: High risk medulloblastoma-preoperative

chemotherapy regimens indicated that residual tumor less than $1.5 \mathrm{~cm}^{2}$ was associated with improvement in 5-year progression free survival (PFS) of $>20 \%$ in patients with M0 disease and an $11 \%$ difference for all patients irrespective of all other factors. ${ }^{[19]}$ Subsequent studies have questioned this premise when taking into account biological factors. A recent retrospective analysis of 787 patients demonstrated that the benefit of extent of resection is largely attenuated after taking into account molecular subtype and not significant when comparing STR $\left(>1.5 \mathrm{~cm}^{2}\right)$ versus NTR $\left(<1.5 \mathrm{~cm}^{2}\right)$ or GTR versus NTR. For tumors involving the brainstem, investigators found no difference in outcome between GTR and residual tumor $<1.5 \mathrm{~cm}^{2[6]}$ Aggressive resection of brainstem disease with potential of high morbidity is, therefore, not warranted in view of the sensitivity of the tumor to radiation and chemotherapy. 
Children typically present with features of raised ICP due to obstructive hydrocephalus. However, routine preoperative ventriculoperitoneal shunt should be avoided as definitive surgical resection readily relieves the obstruction. Ventricular diversion if needed may be achieved by EVD or an endoscopic third ventriculostomy ${ }^{[20,21]}$ taking care to avoid rapid decompression of the ventricles and overdrainage. Corticosteroids may be required in the preoperative period. Dexamethasone in a loading dose of $0.5-1 \mathrm{mg} / \mathrm{kg}$ intravenously (maximum dose $10 \mathrm{mg}$ ) followed by $0.25-0.5 \mathrm{mg} / \mathrm{kg} /$ day can be given in divided doses.

It is imperative that wherever feasible, screening of the spine should be is done prior to surgery. If not feasible preoperatively, it should be acquired postoperatively 2-3 weeks after surgery to reduce the chance of erroneous interpretation consequent to postoperative enhancement of spinal leptomeninges. ${ }^{[22]}$

All patients must undergo lumbar puncture and CSF cytopathology to evaluate for dissemination 2-3 weeks' following surgery. Intraoperative samples taken from ventricles do not suffice for this purpose. ${ }^{[23]}$

The postoperative clinical course can be complicated by the posterior fossa syndrome or cerebellar mutism syndrome in $8 \%-24 \%$ of infratentorial tumor resections. It usually presents in the first 2 days following surgery and is characterized by a triad of cerebellar mutism, ataxia/axial hypotonia, and irritability and emotional lability. These children are commonly apathetic, and/or hypokinetic. While pathophysiology is poorly understood, possible mechanisms include disruption of the dentate-thalamo-cortical pathway, vermian injury, postoperative vasospasm, axonal injury, and neuronal dysfunction. ${ }^{[24,25]}$

\section{Radiation}

Postoperative adjuvant RT is an integral component of therapy for all children above 3 years of age. In view of the high propensity of the tumor to develop leptomeningeal disease, CSI followed by boost irradiation of the tumor bed/ posterior fossa is recommended to achieve adequate disease control preferably delivered from a linear accelerator.

The recommended dose is 54-55 Gy delivered over 6-6.5 weeks using conventional fractionation. The dose for CSI for rigorously staged standard risk MBs is $23.4 \mathrm{~Gy}$ in 13 fractions followed by tumor-bed boost (30.6 Gy in 17 fractions) to a total tumor-bed dose of 54 Gy in 30 fractions over 6 weeks. Such therapy in conjunction with adjuvant multiagent systemic chemotherapy results in excellent long-term survival outcomes ${ }^{[26,27]}$ but with reduced neurocognitive and endocrinological sequelae compared to the higher doses of CSI. High risk disease is treated with full-dose CSI (35-36 Gy in 20-21 fractions) plus posterior fossa boost (18-19.8 Gy in 10-11 fractions) to a total tumor dose of 54-55 Gy in 30-32 fractions over 6-6.5 weeks. Patients with diffuse leptomeningeal dissemination should receive extended dose CSI (39.6-40 Gy in 22-24 fractions) plus entire posterior fossa boost (14.4 Gy in 8 fractions). A boost of 5.4-9 Gy in 3-5 fractions to focal nodular metastatic deposits in the brain and/or spine can be delivered concurrently during posterior fossa boost irradiation.

Adjuvant RT should ideally begin within 4 weeks of surgery, but definitely within 6 weeks post surgery. Routine use of steroids and GCSF is avoided but may be required.

Proton therapy, where available, may be preferred over photons primarily to limit the burden of both short and long-term effects attributable to radiation. ${ }^{[28,29]}$ However, lack of access to this modality limits it to a very small minority of patients in our setting.

Conventionally, radiosensitizing chemotherapy with concurrent weekly vincristine has been used extensively and is tolerated well in young children. However, there is limited evidence of its role and in view of the significant morbidity attributable to neuropathy especially in older children and adolescents, a number of current protocols omit weekly vincristine in this cohort.

For "high risk" lesions addition of daily carboplatin to weekly vincristine has been evaluated. ${ }^{[30]}$ Carboplatin is potent radiosensitizer and this approach appears to enhance outcomes for this patients with metastatic disease. However, it is important to remember that delivering uninterrupted RT is more important than adding Carboplatin as a radiosensitizer. The ACNS0332 Phase III data suggest no benefit in response or survival with this strategy.

It is ideal to document neurocognitive, endocrinal, and auditory status prior to initiation of adjuvant therapy to establish a baseline for future comparisons.

\section{Chemotherapy}

Adjuvant chemotherapy, in the current era is an integral part of the management of $\mathrm{MB}$ in children. ${ }^{[26,31]}$ For children above 3 years, adjuvant chemotherapy should ideally start within 4 weeks of radiation, but definitely within 6 weeks. This period is required for hematological recovery. Neuraxial imaging should be done for re-assessment of the disease status prior to the initiation of adjuvant chemotherapy. A total of 6-8 cycles of adjuvant chemotherapy should be administered generally cycled at 3-6 weekly intervals depending on the regimen used. A number of regimens may contain platinum and it is therefore prudent to monitor with audiometry during the treatment as well. The risk of ototoxicity is higher if cisplatin dose in individual cycle exceeds 100 $\mathrm{mg} / \mathrm{m}^{2}$ or cumulative doses exceed $300 \mathrm{mg} / \mathrm{m}^{2}{ }^{[32]}$ The current evidence indicates that it may not be necessary to deliver cumulative Cisplatin doses of upto $600 \mathrm{mg} / \mathrm{m}^{2}$ scheduled in older protocols. Lower doses may be equally effective. ${ }^{[33]}$ Attempt should be made to deliver optimal 


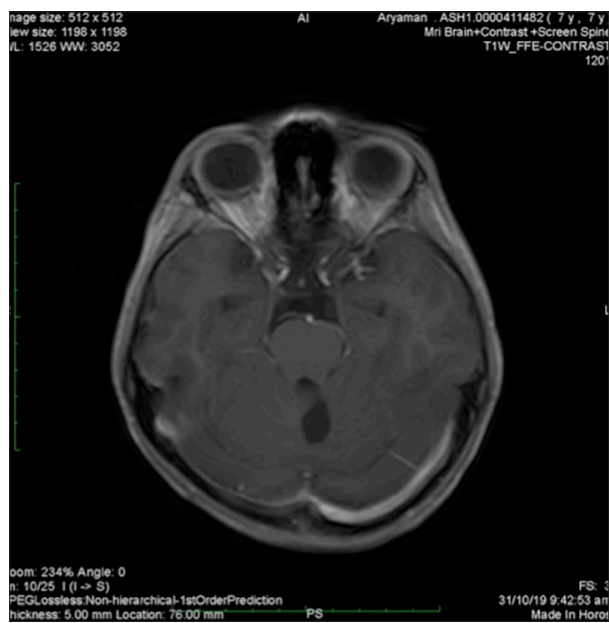

Figure 4: High risk medulloblastoma-postradiation

cumulative doses of cyclophosphamide $\left(12 \mathrm{~g} / \mathrm{m}^{2}\right)$ and Cisplatin $\left(300-400 \mathrm{mg} / \mathrm{m}^{2}\right) \cdot{ }^{[31]}$ For children under 3 years of age, adjuvant therapy comprises of primarily chemotherapy with delayed or no radiation to spare young children from devastating late effects. ${ }^{[34-38]}$

High-dose chemotherapy with stem cell rescue has been evaluated for MB. It is feasible, and may offer an advantage in patients with metastatic or recurrent disease. It offers no survival advantage in older children receiving CSI and in LMIC may be reserved for patients with recurrent disease only. Prior CSI may render stem cell mobilization difficult but plerixafor can overcome this. ${ }^{[39-41]}$

The Milan strategy for metastatic MB incorporated intensive chemotherapy with myeloablative chemotherapy in selected cases along with hyperfractionated radiation yielded excellent outcomes. However, similar results could not be achieved by other centres with this strategy and it is no longer being actively pursued. ${ }^{[42]}$

\section{Case 3}

\section{Medulloblastoma in $<3$ years old}

A 21-month-old child presented with irritability, and increasing head size for 3 months. Neuroimaging revealed a large $4^{\text {th }}$ ventricular tumor with marked hydrocephlus [Figure 5]. He underwent midline suboccipital craniotomy with microsurgical gross total excision of the tumor with duraplasty [Figure 6]. The right frontal $3^{\text {rd }}$ ventriculostomy was done prior to tumour excision. Histology confirmed MB of the desmoplastic nodular type. Molecular studies confirmed $\mathrm{SHH}$ activated variant. Postoperative imaging revealed no residual disease.

The patient was commenced on systemic chemotherapy as per HIT SKK regimen with 12 cycles comprising of carboplatin, methotrexate, cyclophosphamide, and etoposide. Ommaya reservoir was inserted to deliver ventricular methotrexate in each cycle to a total of 32 doses. ${ }^{[35]}$

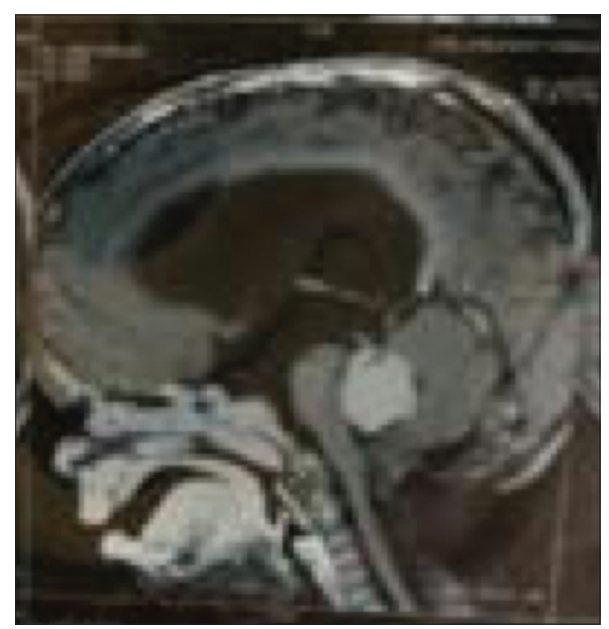

Figure 5: Meulloblastoma in $<3$ years old

The patient was reassessed after every 4 cycles to confirm continuing remission. Decision was taken to omit radiation altogether. The patient continues to be free of disease 2 years' post completion of therapy.

Up to one-third of cases of MB occur during the first 3 years of life. Historically, the survival rates for this cohort have been poor and did not exceed $25 \%-45 \%$ until the past decade. The relatively unfavorable prognosis may partly be explained by more frequent occurrence of metastases and the different biology of $\mathrm{MB}$ in young children. Further, the immature brain is particularly susceptible to radiotherapy-induced neurocognitive deficits warranting the omission of this modality.

Treatment strategies for young children with MB have been aimed at improving survival whilst limiting neurocognitive sequelae. Treatment approaches have been focused on delaying or omitting radiotherapy using conventional systemic chemotherapy incorporating high dose and intra-ventricular methotrexate, high-dose chemotherapy with autologous stem cell rescue and tandem transplant following induction chemotherapy with/without high dose methotrexate. ${ }^{[34-38]}$

The pilot trial HIT-SKK'87 confirmed that postoperative chemotherapy may successfully delay the start of radiotherapy. ${ }^{[34]}$ Intraventricular methotrexate was introduced as a substitute for radiotherapy in the subsequent HIT-SKK'92 trial and HIT 2000 study. ${ }^{[35]}$ If complete remission was achieved, survival rates, especially for young patients with DMB (5-year PFS and OS 85\% and 95\% respectively were very favorable. Neurocognitive deficits were reduced as compared with the HIT-SKK' 87 trial. The estimated survival rates for the entire cohort (5-year EFS rate, $57 \%+8 \%$; 5-year OS rate, $80 \%+6 \%$ ) compared favorably with results of older studies.

Exclusively chemotherapy-based approach as first-line treatment may contribute to improved salvage strategies at relapse: while $50 \%$ relapses were successfully salvaged in 


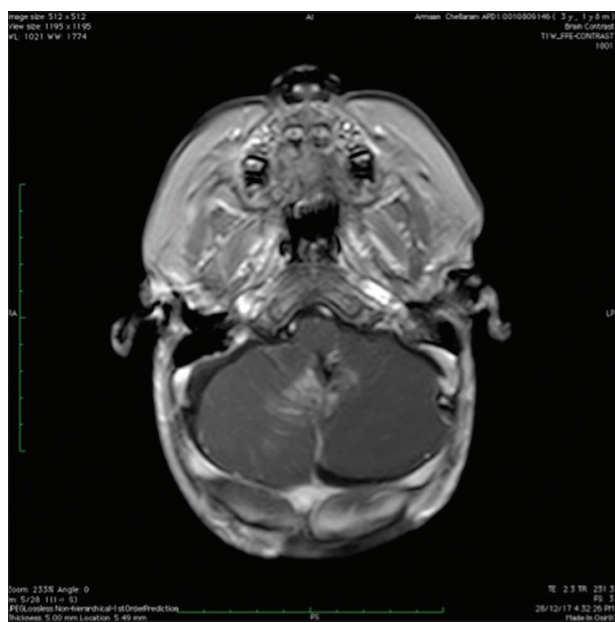

Figure 6: Medulloblastoma in $<3$ years old-postoperative

HIT-SKK' 92, only 1 of 10 treated children were salvaged in HIT-SKK' 87 trial.

These studies also confirmed that histology is a strong prognostic factor in this age group. This is important as DMB/MBEN account for $40 \%$ of cases in this cohort. ${ }^{[38]}$ Gross total resection appears to be more common and feasible for patients with DMB or MBEN. All infants with $\mathrm{SHH} \mathrm{MB}$ have a favorable prognosis regardless of histology.

For children with non-DMB/non-MBEN, for which predominantly local relapses lead to less favorable survival rates, local radio-therapy has been introduced after chemotherapy since 2006. However, infants with non-SHH MB mainly belong to Subgroup 3 and usually succumb to metastatic disease, and focal RT approaches have failed to improve outcomes in this cohort. The best results till date are with the COG0334 protocol on the high-dose methotrexate arm added to the 99,703 tandem transplant backbone (to be published). Therefore, careful consideration is needed prior to offering focal RT to infant with subgroup $3 \mathrm{MB}$ as this may not have any survival benefit.

The SJYC07 Phase II $^{[43]}$ study offered molecularly driven risk adapted therapy for young children with MB. The study identified a good responder $\mathrm{SHH}$ subtype (iSHH-II) that exhibits excellent progression-free survival in the absence of radiation, intra-ventricular or high-dose chemotherapy in contrast to the poor responder iSHH-I that has much inferior outcomes.

Recent data incorporating genomics now mandates that future approaches for young children will increasingly incorporate molecularly driven, risk-adapted approaches.

\section{Follow-up after treatment}

Surveillance imaging during and after treatment aimed at detecting recurrent disease at an early stage in asymptomatic patients, has been arbitrarily determined and not been shown to influence survival. Isolated spinal recurrences are infrequent and follow-up imaging must be tailored based on risk assignment. ${ }^{[4]}$ It is imperative that strategies for follow-up incorporate multidisciplinary review inclusive of audiological, neuropsychological and endocrine evaluations.

\section{Case 4}

\section{Recurrent medulloblastoma}

A 7-year-old young girl had been diagnosed with classical $\mathrm{MB}$ at 5 years of age. She had undergone gross total resection and received adjuvant radiotherapy and chemotherapy. One year after completion of chemotherapy, she presented with recurrent disease locally. Surgical resection was attempted followed by high-dose chemotherapy with stem cell rescue. Unfortunately, despite this the disease progressed and she succumbed.

Options for recurrent disease are currently limited and largely unsuccessful. The pattern of failure is likely subgroup-specific and can guide management decisions. Eg: Group 3 and Group 4 usually have distant recurrence after RT-based treatment. SHH-MB may recur both locally and with distant metastasis. Patients with germline conditions are also at risk of second malignancies which need to be recognized and appropriately managed.

Surgery, re-irradiation, and chemotherapy regimens including myeloablative chemotherapy have been explored with poor results. ${ }^{[45,46]}$ A proportion of infants treated previously with only chemotherapy may be salvaged with CSI with profound neurocognitive sequelae. Bevacizumab and irinotecan with or without temozolomide has been shown to give objective response rate and is well tolerated. ${ }^{[47]}$

An evolving alternative approach is to target tumor angiogenesis with metronomic therapy incorporating bevacizumab, thalidomide, celecoxib, fenofibrate, etoposide, and cyclophosphamide and additional intraventricular therapy (etoposide and liposomal cytarabine) which can be delivered with manageable toxicity. ${ }^{[48]}$

\section{Future strategies}

Specifically targeted chemotherapies targeting oncogenic pathways are a promising future application of molecular subgrouping. Currently, molecularly targeted agents for each of the four molecular subgroups are being evaluated in preclinical and clinical models. The most well-studied of these is Vismodegib, that has demonstrated some utility in both preclinical and clinical models and may be an option in adolescents and older children. ${ }^{[49]}$

Alternative strategies include sensitization of MB tumor cells to chemotherapeutic treatment. Thiostrepton, an antagonist of FOXM1 (an oncogene known to be 
upregulated in a variety of malignancies), was shown to sensitize MB cells to cisplatin in vitro. ${ }^{[50]}$

In our setting, future strategies must focus on improving access to molecular classification which is the cornerstone for improving outcomes and limiting toxicity. For example, infant $\mathrm{SHH}$ even with metastasis may be curable using chemotherapy only approaches and WNT tumors may be offered reduced dose CSI. It is therefore, imperative that ongoing efforts in LMIC focus on this. Till genomic profiling becomes more widely available, validation, and incorporation of IHC and radiogenomics could play a major role in delievering risk-adapted treatments aimed at optimizing outcomes and limiting toxicity.

\section{Summary and Recommendations}

- Optimal treatment of MB can yield high cure rates even in countries with limited resources

- The current risk stratification incorporates clinical, histopathological and molecular characteristics

- Molecular characterization, while of immense prognostic and therapeutic significance may not be feasible for the vast majority of patients in our setting

- Immunohistochemistry-based approach to molecular characterization may offer a pragmatic approach

- Optimal resection is the cornerstone of treatment. However, in view of the radio and chemo-sensitivity of the tumor, heroic attempts at complete resection associated with neurological morbidity are unwarranted

- Placement of VP shunts is not required and temporary diversion measures should suffice in cases with marked hydrocephalus

- Radiation is an essential part of treatment in older children. It should be or omitted/deferred in younger children wherever feasible. Tumor bed boost is equivalent to posterior fossa boost

- Current evidence supports adjuvant chemotherapy in all patients for 6-8 cycles. High-dose chemotherapy may offer an advantage in salvage settings

- Ongoing approaches are focused on improving outcomes for high risk disease and limiting the late effects associated with treatment

- For LMIC these should be focused on improving access to molecular stratification.

\section{Financial support and sponsorship}

Nil.

\section{Conflicts of interest}

There are no conflicts of interest.

\section{References}

1. Taylor MD, Northcott PA, Korshunov A, Remke M, Cho YJ, Clifford SC, et al. Molecular subgroups of medulloblastoma: The current consensus. Acta Neuropathol 2012;123:465-72.
2. Ramaswamy V, Remke M, Bouffet E, Bailey S, Clifford SC, Doz F, et al. Risk stratification of childhood medulloblastoma in the molecular era: The current consensus. Acta Neuropathol 2016;131:821-31.

3. Louis DN, Perry A, Reifenberger G, von Deimling A, Figarella-Branger D, Cavenee WK, et al. The 2016 World Health Organization Classification of Tumors of the Central Nervous System: A summary. Acta Neuropathol 2016;131:803-20.

4. Gupta T, Sarkar C, Rajshekhar V, Chatterjee S, Shirsat N, Muzumdar D, et al. Indian Society of Neuro-Oncology consensus guidelines for the contemporary management of medulloblastoma. Neurol India 2017;65:315-32.

5. Parkes J, Hendricks M, Ssenyonga P, Mugamba J, Molyneux E, Schouten-van Meeteren A, et al. SIOP PODC adapted treatment recommendations for standard-risk medulloblastoma in low and middle income settings. Pediatr Blood Cancer 2015;62:553-64.

6. Thompson EM, Hielscher T, Bouffet E, Remke M, Luu B, Gururangan $\mathrm{S}$, et al. Prognostic value of medulloblastoma extent of resection after accounting for molecular subgroup: A retrospective integrated clinical and molecular analysis. Lancet Oncol 2016;17:484-95.

7. Pfister S, Remke M, Benner A, Mendrzyk F, Toedt G, Felsberg $\mathrm{J}$, et al. Outcome prediction in pediatric medulloblastoma based on DNA copy-number aberrations of chromosomes $6 \mathrm{q}$ and $17 \mathrm{q}$ and the MYC and MYCN loci. J Clin Oncol 2009;27:1627-36.

8. Goschzik T, Schwalbe EC, Hicks D, Smith A, Zur Muehlen A, Figarella-Branger D, et al. Prognostic effect of whole chromosomal aberration signatures in standard-risk, non-WNT/non-SHH medulloblastoma: A retrospective, molecular analysis of the HIT-SIOP PNET 4 trial. Lancet Oncol 2018;19:1602-16.

9. Waszak SM, Northcott PA, Buchhalter I, Robinson GW, Sutter C, Groebner S, et al. Spectrum and prevalence of genetic predisposition in medulloblastoma: A retrospective genetic study and prospective validation in a clinical trial cohort. Lancet Oncol 2018;19:785-98.8.

10. Das A, Ramaswamy V. Less treatment for Wing less medulloblastoma: Germline data re-emphasize this. Neuro Oncol 2020;22:7-9.

11. Tabori U, Baskin B, Shago M, Alon N, Taylor MD, Ray PN, et al. Universal poor survival in children with medulloblastoma harboring somatic TP53 mutations. J Clin Oncol 2010;28:1345-50.

12. Northcott PA, Hielscher T, Dubuc A, Mack S, Shih D, Remke M, et al. Pediatric and adult sonic hedgehog medulloblastomas are clinically and molecularly distinct. Acta Neuropathol 2011;122:231-40.

13. Kaur K, Kakkar A, Kumar A, Mallick S, Julka PK, Gupta D, et al. Integrating molecular subclassification of medulloblastomas into routine clinical practice: A simplified approach. Brain Pathol 2016;26:334-43.

14. Iv M, Zhou M, Shpanskaya K, Perreault S, Wang Z, Tranvinh E, et al. MR imaging-based radiomic signatures of distinct molecular subgroups of medulloblastoma. AJNR Am J Neuroradiol 2019;40:154-61.

15. Dasgupta A, Gupta T, Pungavkar S, Shirsat N, Epari S, Chinnaswamy G, et al. Nomograms based on preoperative multiparametric magnetic resonance imaging for prediction of molecular subgrouping in medulloblastoma: Results from a radiogenomics study of 111 patients. Neuro Oncol 2019;21:115-24. 
16. Kool M, Korshunov A, Remke M, Jones DT, Schlanstein M, Northcott PA, et al. Molecular subgroups of medulloblastoma: An international meta-analysis of transcriptome, genetic aberrations, and clinical data of WNT, SHH, Group 3, and Group 4 medulloblastomas. Acta Neuropathol 2012;123:473-84.

17. Brown HG, Kepner JL, Perlman EJ, Friedman HS, Strother DR, Duffner PK, et al. "Large cell/anaplastic" medulloblastomas: A Pediatric Oncology Group Study. J Neuropathol Exp Neurol 2000;59:857-65.

18. Huang PI, Lin SC, Lee YY, Ho DM, Guo WY, Chang KP, et al. Large cell/anaplastic medulloblastoma is associated with poor prognosis-a retrospective analysis at a single institute. Childs Nerv Syst 2017;33:1285-94.

19. Albright AL, Wisoff JH, Zeltzer PM, Boyett JM, Rorke LB, Stanley P. Effects of medulloblastoma resections on outcome in children: A report from the Children's Cancer Group. Neurosurgery 1996;38:265-71.

20. Fritsch MJ, Doerner L, Kienke S, Mehdorn HM. Hydrocephalus in children with posterior fossa tumors: Role of endoscopic third ventriculostomy. J Neurosurg 2005;103:40-2.

21. Muzumdar D, Deshpande A, Kumar R, Sharma A, Goel N, Dange $\mathrm{N}$, et al. Medulloblastoma in childhood-King Edward Memorial hospital surgical experience and review: Comparative analysis of the case series of 365 patients. J Pediatr Neurosci 2011;6:S78-85.

22. Meyers SP, Wildenhain SL, Chang JK, Bourekas EC, Beattie PF, Korones DN, et al. Postoperative evaluation for disseminated medulloblastoma involving the spine: Contrast-enhance MRI findings, CSF cytologic analysis, timing of disease recurrence, and patient outcomes. AJNR Am J Neurradiol 2000;21:1757-65.

23. Terterov S, Krieger MD, Bowen I, McComb JG. Evaluation of intracranial cerebrospinal fluid cytology in staging pediatric medulloblastomas, primitive neuroectodermal tumors, and ependymomas. J Neurosurg Pediatr 2010;6:131-6.

24. Schreiber JE, Palmer SL, Conklin HM, Mabott DJ, Swain MA, Bonner MJ, et al. Posterior fossa syndrome and long-term neuropsychological outcomes among children treated for medulloblastoma on a multi-institutional, prospective study. Neuro Oncol 2017;19:1673-82.

25. Lanier JC, Abrams AN. Posterior fossa syndrome: Review of the behavioral and emotional aspects in pediatric cancer patients. Cancer 2017;123:551-9.

26. Packer RJ, Gajjar A, Vezina G, Rorke-Adams L, Burger PC, Robertson PL, et al. Phase III study of craniospinal irradiation followed by adjuvant chemotherapy for newly diagnosed average-risk medulloblastoma. J Clin Oncol 2006;24:4202-8.

27. Thomas PR, Deutsch M, Kepner JL, Boyett JM, Krischer J, Aronin $\mathrm{P}$, et al. Low-stage medulloblastoma: Final analysis of trial comparing standard-dose with reduced-dose neuraxis irradiation. J Clin Oncol 2000;18:3004-11.

28. Grosshans DR. Proton therapy for pediatric medulloblastoma. Lancet Oncol 2016;17:258-9.

29. Sreeraman R, Indelicato DJ. Proton therapy for the treatment of children with CNS malignancies. CNS Oncol 2014;3:149-58.

30. Jakacki RI, Burger PC, Zhou T, Holmes EJ, Kocak M, Onar A, et al. Outcome of children with metastatic medulloblastoma treated with carboplatin during craniospinal radiotherapy: A children's oncology group phase I/II study. J Clin Oncol 2012;30:2648-53.

31. Michels EM, Schouten-Van Meeteren AY, Doz F, Janssens GO, van Dalen EC. Chemotherapy for children with medulloblastoma. Cochrane Database Syst Rev 2015;1:CD006678.

32. Li Y, Womer RB, Silber JH. Predicting cisplatin ototoxicity in children: The influence of age and the cumulative dose. Eur $\mathrm{J}$ Cancer 2004;40:2445-51.

33. Nageswara Rao AA, Wallace DJ, Billups C, Boyett JM, Gajjar A, Packer RJ, et al. Cumulative cisplatin dose is not associated with event-free or overall survival in children with newly diagnosed average-risk medulloblastoma treated with cisplatin based adjuvant chemotherapy: Report from the Children's Oncology Group. Pediatr Blood Cancer 2014;61:102-6.

34. Kuhl J, Beck J, Bode U. Delayed radiation therapy after postoperative chemotherapy in children less than 3 years of age 14 with medulloblastoma: Results of the trial HIT-SKK 87 study and preliminary results of the pilot trial HIT-SKK 92. Med Pediatr 15 Oncol 1995;25 Suppl:250.

35. Rutkowski S, Bode $\mathrm{U}$, Dienlein $\mathrm{F}$, Ottensmeier $\mathrm{H}$, Warmuth-Metz M, Sorensen N, et al. Treatment of early childhood medulloblastoma by postoperative chemotherapy alone. N Engl J Med 2005;352:978-86.

36. Rutkowski S. Current treatment approaches to early childhood medulloblastoma. Expert Rev Neurother 2006;6:1211-21.

37. Rutkowski S, Gerber NU, von Hoff K, Gnekow A, Bode U, Graf N, et al. Treatment of early childhood medulloblastoma by postoperative chemotherapy and deferred radiotherapy. Neuro Oncol 2009;11:201-10.

38. Von bueren AO, von Hoff $\mathrm{K}$, petsch $\mathrm{T}$, Gerber NU, Warmuth-Metz M, Deinlein F, et al. Treatment of young children with localized medulloblastoma by chemotherapy alone: Results of the prospective, multicenter trial HIT 2000 confirming the prognostic impact of histology. Neuro Oncol 2011;13:669-79.

39. Perez-Martinez A, Lassaletta A, Gonzalez-Vincent M, Sevilla J, Diaz MA, Madero L. High dose chemotherapy with autologous stem cell rescue for children with high risk and recurrent medulloblastoma and supratentorial primitive neuroectodermal tumors. J Neurooncol 2005;71:33-8.

40. Gajjar A, Chintagumpala M, Ashley D, Kellie S, Kun LE, Merchant TE, et al. Risk-adapted craniospinal radiotherapy followed by high-dose chemotherapy and stem-cell rescue in children with newly diagnosed medulloblastoma (St. Jude Medulloblastoma-96): Long-term results from a prospective, multicentre trial. Lancet Oncol 2006;7:813-20.

41. Sung KW, Yoo KH, Cho EJ, Koo HH, Lim DH, Shin HJ, et al. High-dose chemotherapy and autologous stem cell rescue in children with newly diagnosed high-risk or relapsed medulloblastoma or supratentorial primitive neuroectodermal tumor. Pediatr Blood Cancer 2007;48:408-15.

42. Gandola L, Massimino M, Cefalo G, Solero C, Spreafico F, Pecori E, et al. Hyperfractionated accelerated radiotherapy in the Milan strategy for metastatic medulloblastoma. J Clin Oncol 2009;27:566-71.

43. Robinson GW, Rudneva VA, Buchhalter I, Billups C, Waszak SM, Smith KS, et al. Risk-adapted therapy for young children with medulloblastoma (SJYC07): Therapeutic and molecular outcomes from a multicentre, phase 2 trial. Lancet Oncol 2018;19:768-84.

44. Perreault S, Lober RM, Carret AS, Zhang G, Hershon L, Decarie JC, et al. Surveillance imaging in children with malignant CNS tumors: Low yield of spine MRI. J Neurooncol 2014;116:617-23.

45. Gajjar A, Pizer B. Role of high-dose chemotherapy for recurrent medulloblastoma and other CNS primitive neuroectodermal tumors. Pediatric Blood Cancer 2010;54:649-51. 
46. Sabel M, Fleischhack G, Tippelt S, Gustafsson G, Doz F, Kortmann R, et al. Relapse patterns and outcome after relapse in standard risk medulloblastoma: A report from the HIT-SIOP-PNET4 study. J Neurooncol 2016;129:515-24.

47. Bonney PA, Santucci JA, Maurer AJ, Sughrue ME, McNall-Knapp RY, Battiste JD. Dramatic response to temozolomide, irinotecan, and bevacizumab for recurrent medulloblastoma with widespread osseous metastases. J Clin Neurosci 2016;26:161-3.

48. Peyrl A, Chocholous M, Kieran MW, Azizi AA, Prucker C, Czech T, et al. Antiangiogenic metronomic therapy for children with recurrent embryonal brain tumors. Pediatr Blood Cancer 2012;59:511-7.

49. Petrirena GJ, Masliah-Planchon J, Sala Q, Pourroy B, Frappaz D, Tabouret E, et al. Recurrent extraneural sonic hedgehog medulloblastoma exhibiting sustained response to vismodegib and temozolomide monotherapies and inter-metastatic molecular heterogeneity at progression. Oncotarget 2018;9:10175-83.

50. Lin J, Zheng Y, Chen K, Huang Z, Wu X, Zhang N. Inhibition of FOXMI by thiostrepton sensitizes medulloblastoma to the effects of chemotherapy. Oncol Rep 2013;30:1739-44. 